BNL - 73207-2004-CP

$\mathrm{ATF}-04 \mathrm{C}$ Experiments on Laser and e-Beam Transport and
Interaction in a Plasma Channel

I.V. Pogorelsky ${ }^{a}$, I.V. Pavlishin ${ }^{a}, \mathrm{I}_{\text {. Ben-Zvi }}{ }^{a}$, V. Yakimenko ${ }^{a}$, T. Kumita ${ }^{b}$, Y. Kamiya ${ }^{b}$, A. Zigler ${ }^{c}$, A. Diublov ${ }^{d}$, N. Andreev ${ }^{e}$, N. Bobrova ${ }^{f}$ and P. Sasorov ${ }^{f}$

a Accelerator Test Facility, Brookhaven National Laboratory, 820, Upton, NY 11973, USA

${ }^{b}$ Physics Department, Tokyo Metropolitan University, Japan

${ }^{c}$ Hebrew University, Jerusalem, Israel

${ }^{d}$ Optoel Co, St. Petersburg, Russia

- Inst. High Density Energy, Moscow, Russia

${ }^{f}$ Inst. Theoretical and Experimental Phys., Moscow, Russia

September 2004

Presented at " $11^{\text {th }}$ Advanced Accelerator Concepts Workshop (AAC2004)", Stony Brook, New York, June 21-26, 2004. 


\section{DISCLAIMER}

This report was prepared as an account of work sponsored by an agency of the United States Government. Neither the United States Government nor any agency thereof, nor any of their employees, nor any of their contractors, subcontractors or their employees, makes any warranty, express or implied, or assumes any legal liability or responsibility for the accuracy, completeness, or any third party's use or the results of such use of any information, apparatus, product, or process disclosed, or represents that its use would not infringe privately owned rights. Reference herein to any specific commercial product, process, or service by trade name, trademark, manufacturer, or otherwise, does not necessarily constitute or imply its endorsement, recommendation, or favoring by the United States Government or any agency thereof or its contractors or subcontractors. The views and opinions of authors expressed herein do not necessarily state or reflect those of the United States Government or any agency thereof. 


\title{
Experiments on Laser and e-Beam Transport and Interaction in a Plasma Channel
}

\author{
I.V. Pogorelsky ${ }^{a}$, I.V. Pavlishin ${ }^{a}$, I. Ben-Zvi ${ }^{a}$, V. Yakimenko ${ }^{a}$, \\ T. Kumita ${ }^{b}$, Y. Kamiya ${ }^{b}$, A. Zigler ${ }^{c}$, A. Diublov $^{d}$, N. Andreev ${ }^{e}$, \\ N. Bobrova ${ }^{f}$ and P. Sasorov ${ }^{f}$ \\ ${ }^{a}$ Accelerator Test Facility, Brookhaven National Laboratory, 820, Upton, NY 11973, USA \\ ${ }^{b}$ Physics Department, Tokyo Metropolitan University, Japan \\ ${ }^{c}$ Hebrew University, Jerusalem, Israel \\ ${ }^{d}$ Optoel Co, St. Petersburg, Russia \\ ${ }^{e}$ Inst. High Density Energy, Moscow, Russia \\ ${ }^{f}$ Inst. Theoretical and Experimental Phys., Moscow, Russia
}

\begin{abstract}
An ablative capillary discharge is installed into a linac beamline and serves as a plasma source for generating and characterizing wakefields. Simultaneously, the electron beam is used as a tool for plasma diagnostics. A high-energy picosecond $\mathrm{CO}_{2}$ laser channeled within the same capillary strongly affects a counterpropagating electron beam. These observations, supported with simulations, suggest the possibility of manipulating relativistic electron beams by steep plasma channels ponderomotively produced by a laser.
\end{abstract}

\section{INTRODUCTION}

A high-power laser guided in a plasma channel is a prospective driver for the nextgeneration high-gradient electron accelerators where electrons pick up momentum from the laser photons moving collinear with the laser beam.

When the laser and electron beam's motion is reversed to counterpropagation, electrons lose energy by generating intense $\mathrm{x}$-ray pulses via Thomson scattering. This process can benefit from optical guiding as well allowing efficient use of relatively long laser pulses [1].

Setting a stage for user's experiments to investigate both electron acceleration and $\mathrm{x}$ ray generation, BNL's ATF, in partnership with Hebrew University, Jerusalem, and Tokyo Metropolitan University initiated R\&D on the e-beam compatible plasma channel suitable for guiding mid-infrared $(\lambda \approx 10 \mu \mathrm{m}) \mathrm{CO}_{2}$ laser radiation that is the ATF's specialty.

The requirements for compatibility with the high-vacuum $\left(10^{-8}\right.$ torr) environment of an electron beamline, setting a plasma density well below the critical value of $10^{19} \mathrm{~cm}^{-3}$ characteristic for the $\lambda=10 \mu \mathrm{m} \mathrm{CO}$ beams, and a relatively wide bore opening to allow the passage of the electron beam, all point towards ablative capillary discharge that runs in a vacuum [2].

We report here the milestones and findings obtained in the course of the ATF's plasma channel project:

- A capillary discharge setup integrated into the electron linac beamline proved to be high-vacuum compatible, and allows precise alignment of the capillary to the incoming electron and laser beams. 
- We established conditions for producing cylindrically profiled plasma on the scale of $\sim 10^{17} \mathrm{~cm}^{-3}$ and demonstrated channeling of an intense $\mathrm{CO}_{2}$ laser beam.

- The capillary discharge served as a plasma source for wakefield studies. The electron beam was used for plasma diagnostics, wakefield generation and probing.

- We observed the effects of e-beam manipulation by the $\mathrm{CO}_{2}$ laser guided in the plasma channel that opens new potentials for e-beam focusing, steering, and electron/laser synchronization.

\section{ABLATIVE CAPILLARY SETUP}

Following the basic design from Hebrew University [2], we constructed a doublestage capillary of the $0.3-1.0 \mathrm{~mm}$ interchangeable bore size made of a plastic such as polyethylene or polypropylene. A high-voltage discharge in a 3-mm long "ignition" capillary segment generates plasma by ablating the inner wall. When the ignition plasma fills a main capillary, which can be several centimeters long, the main storage capacitor fires producing a uniform ablation along the entire capillary's length. Since the capillary's basic chemical composition is $\mathrm{CH}_{2}$, the plasma consists of protons and highly stripped carbon ions.

A plasma density has an axial minimum due to ohmic heating in the discharge. Consequently, the radial distribution of a refraction index defined by a plasma density resembles that observed in a gradient index optical fiber (maximum refraction at the axis). A laser beam can be trapped along the axis of such a plasma fiber by the total internal reflection.

The capillary setup shown schematically in Figure 1 was incorporated into a vacuum interaction cell (Figure 2a) that encloses parabolic laser mirrors with manipulators (Figure 2b). A $17 \mathrm{~mm}$ long polypropylene capillary tube is mounted on a combination of translation and tilt manipulators to facilitate its alignment to the laser- and electronbeams. Standard high-vacuum electrical feedthroughs transmit up to $20 \mathrm{kV}$ of pulsed voltage potentials to the capillary electrodes (schematically shown in Figure 1b).

The insignificant amounts of ablation fumes released by the discharge did not adversely affect the operation of the RF gun and linac.

\section{WAKEFIELD GENERATION AND PROBING}

To begin studying the interaction between the electrons and laser photons channeled inside the capillary, we separately tested e-beam propagation and laser channeling.

Passing a $60 \mathrm{MeV}, 0.5 \mathrm{nC}, 3.5 \mathrm{ps}$ (FWHM) electron beam through a 1-mm-bore capillary, we achieved $80 \%$ of the charge transmission, thereby indicating that the electron beam's size is $\sigma_{b} \leq 200 \mu \mathrm{m}$. Simultaneously, we made a fundamentally important observation. From the energy- and transverse-electron distributions recorded on the electron spectrometer downstream from the interaction cell, we demonstrated phasing between the longitudinal- and transverse-fields in a plasma wake inside the capillary discharge (see Figure 3a) [3]. The maximum in the wake amplitude observed $2 \mu$ s after the discharge (Figure $3 b$ ) is attributed to the plasma's resonance with the time constant of the leading front of the electron bunch. Based on this, we estimated plasma density and 
reconstructed the dynamics of plasma decay in discharge afterglow [3]. This behavior matches hydrodynamic simulations of the plasma outflow through the open ends of the capillary (Figure 4a).

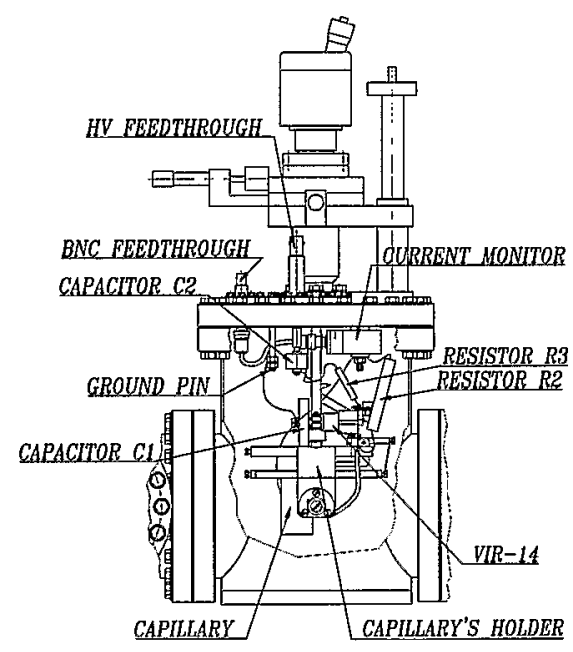

(a)

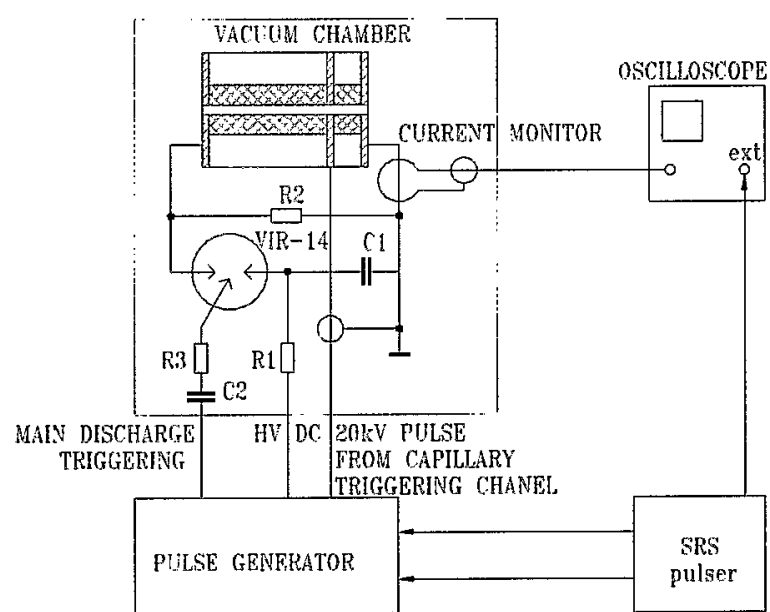

(b)

FIGURE 1. a) Layout of the central section of the interaction cell that contains a discharge capillary; b) principle diagram of the capillary discharge circuit.

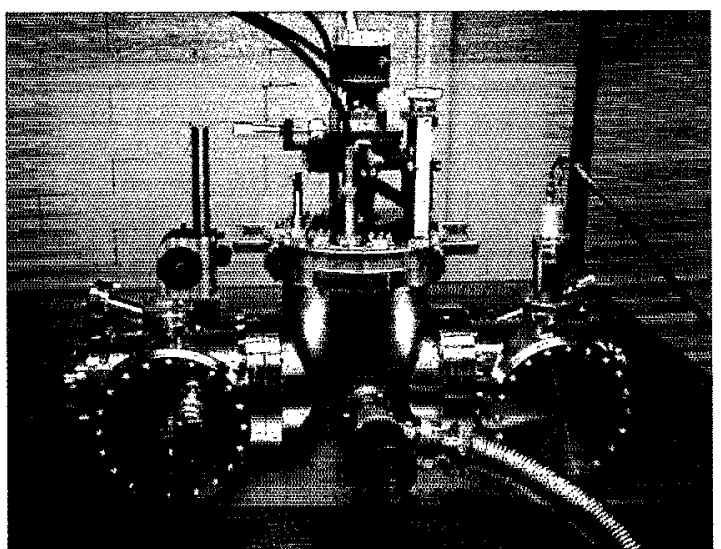

(a)

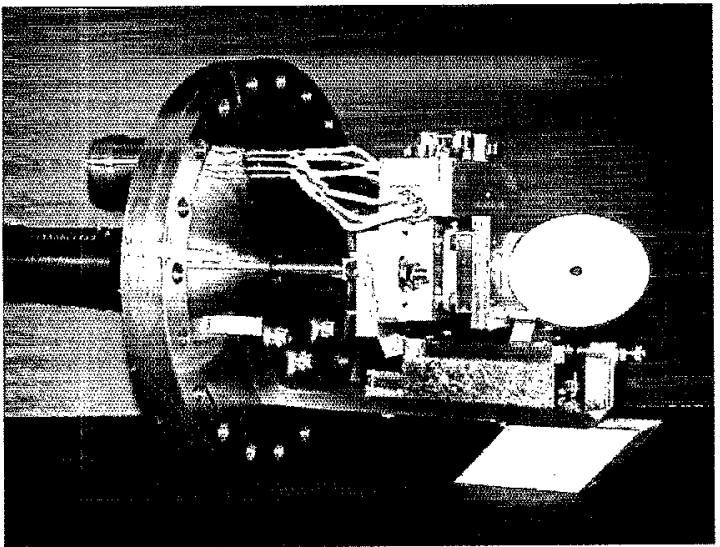

(b)

FIGURE 2. a) Interaction cell; b) laser parabolic mirror prior its mounting inside the cell. 


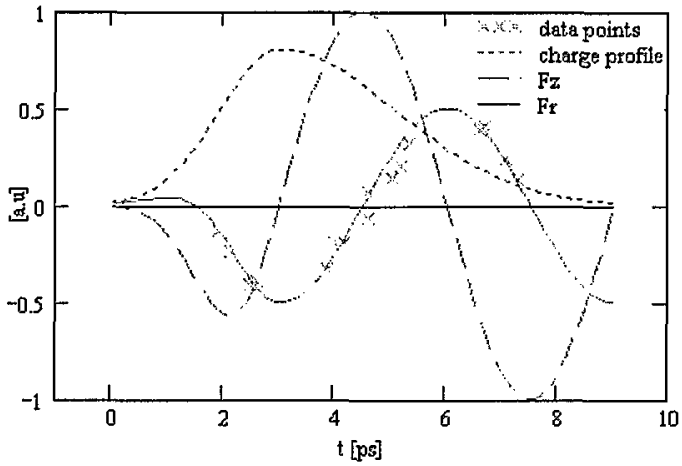

(a)

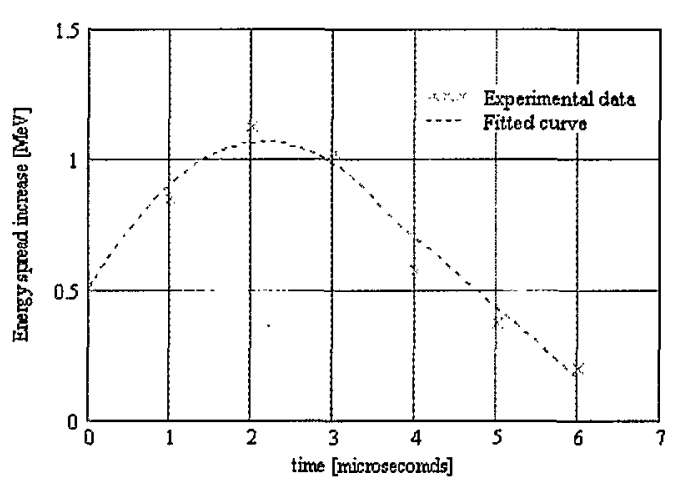

(b)

FIGURE 3. a) Demonstrated phasing between longitudinal and transverse fields in plasma wakes; b) wake amplitude as a function of the time delay after the discharge.

The reported initial plasma wakefield studies have evolved into a user's experiment wherein much stronger fields will be generated inside the $10^{19} \mathrm{~cm}^{-3}$ plasma by the electron beam resonantly microbunched to the $33 \mathrm{fs}$ period, helped by a $\mathrm{CO}_{2}$ laser beam interacting with electrons inside a wiggler [4].

\section{4. $\mathrm{CO}_{2}$ LASER GUIDING IN A PLASMA CHANNEL}

To gain an understanding of the transverse profile of the plasma during the discharge, we used a one-dimensional MHD simulation. Such an approach is valid since the capillary's length is much greater than its diameter [5].

The following dissipative processes were taken into account: electron thermal conductivity, Joule heating, Nernst and Ettinghausen effects, radiation losses, and ion viscosity. To calculate the degree of ionization we assumed that the conditions for local thermodynamic equilibrium are satisfied. The plasma-wall interaction is modeled by considering the wall as a cold neutral gas with atomic number $Z=7$, average atomic weight $A=14$, and density $\rho_{0}=1 \mathrm{~g} / \mathrm{cm}^{3}$. The simulated electron plasma's temperature and density distributions in Figure $4 \mathrm{~b}$ show, correspondingly, the maximum and minimum at the axis due to a combination of wall ablation and current heating.

Solving a set of Maxwell and hydrodynamic equations for plasma electrons and ions simulated channeling of the $\mathrm{CO}_{2}$ laser pulses. Plasma was considered fully ionized and parabollically profiled in the best fit to the distributions in Figure 4b. The input laser pulse duration $180 \mathrm{ps}$ and energy $5 \mathrm{~J}$ that correspond to the ATF experiment conditions. We assumed the temporal and radial Gaussian profile, laser focus radius at the entrance to the channel $w_{0}=120 \mu \mathrm{m}$, normalized laser vector-potential $a_{0}=0.1$. Simulations show pronounced effects of ponderomotive plasma expulsion and ponderomotive self-focusing of the laser beam. 


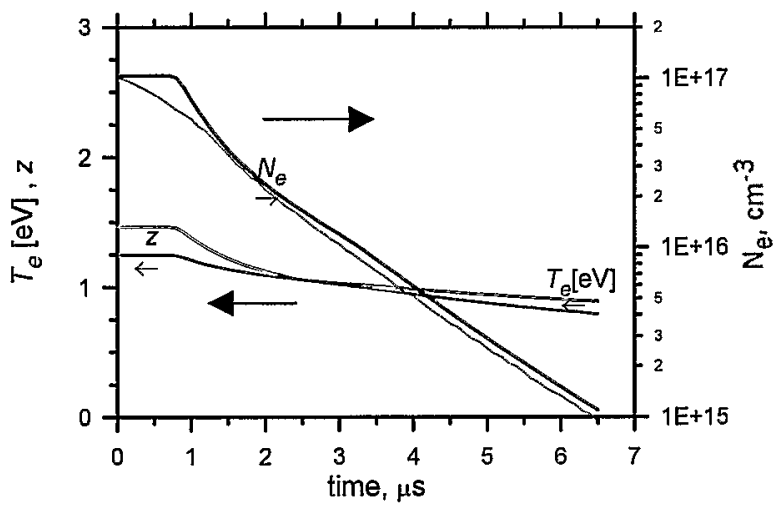

(a)

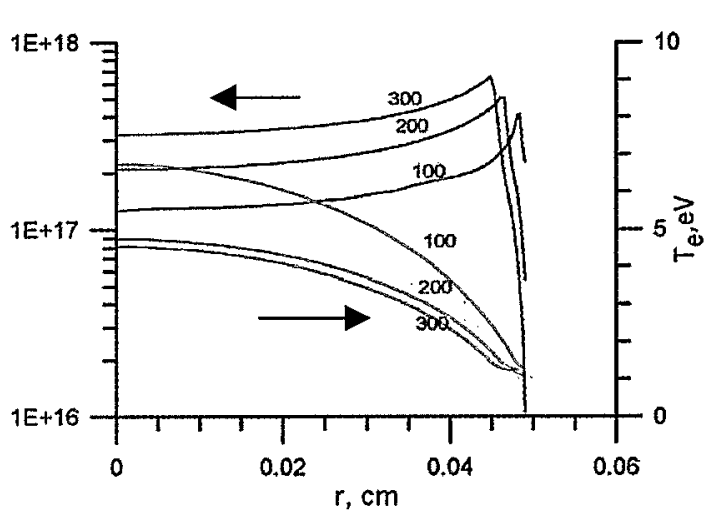

(b)

FIGURE 4. a) Experimental and simulated plasma parameters in the capillary discharge afterglow; b) radial distribution of the electron plasma density and temperature at different moments (measured in nanoseconds) after beginning of the discharge.

The matched condition

$$
R_{c h}=k_{p 0} w_{0}^{2}, \quad k_{p 0}=\omega_{p 0} / c=c^{-1} \sqrt{4 \pi e^{2} n_{e 0} / m_{e}},
$$

where $R_{c h}$ is the radius of the plasma channel and $n_{e \theta}$ is the electron plasma density at the channel axis, ensures the laser pulse propagation along a channel without significant amplitude oscillations and pulse distortions (Figure 5a). At the same time, the plasma density at the channel axis behind the pulse drops approximately three times below the unperturbed density $n_{e 0}$ due to ponderomotive depletion (see Figure $5 b$ ).

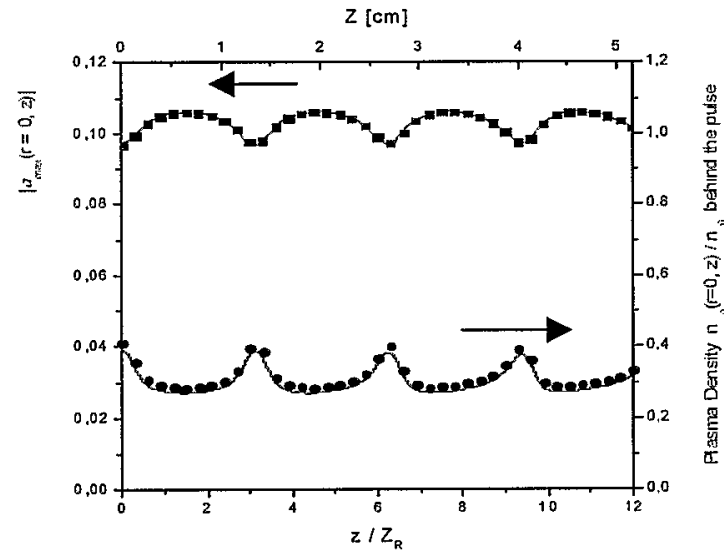

(a)

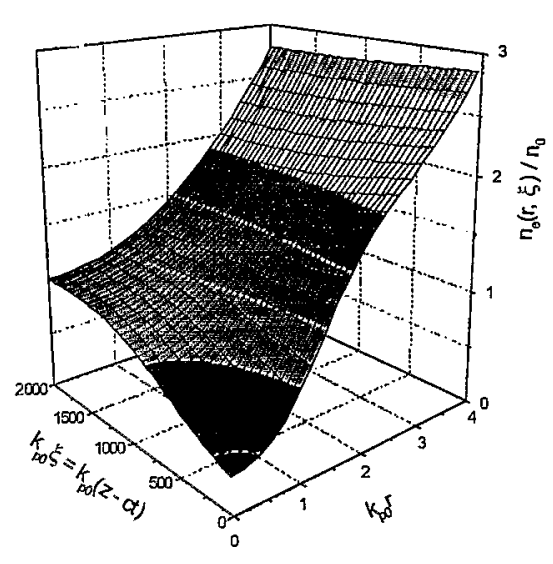

(b)

FIGURE 5. a) Distributions of a normalized laser-pulse intensity and plasma density at the channel axis in the matched condition as functions of the length of propagation along the channel for an arbitrary ion content (not pure hydrogen); b) ponderomotive depletion of plasma density at the channel's exit. 
The importance of the matching condition is illustrated by Figure 6a where the same laser pulse as in Figure 5 was focused into the plasma channel with the radius $R_{c h}=382$ $\mu \mathrm{m}$, which is twice mismatched. The violation of the matching condition results in the increase of oscillations of the laser pulse intensity and the plasma density.

It is instructive to note that somewhat different situation is observed in hydrogen plasma due to higher ion mobility. Here, even at the perfect matching condition, we observe ripples of the laser pulse amplitude and of the plasma density behind the laser pulse (Figure 6b).

In an experiment, the 180-ps (FWHM), 5-J CO 2 laser pulse was focused at the entrance of the $18 \mathrm{~mm}$ long capillary. A magnified output laser beam was imaged with a pyroelectric video camera. Its intensity patterns shown in Figure 7 appear to be the first experimental evidence of guiding of the $10-\mu \mathrm{m}$ laser beam in a plasma channel.

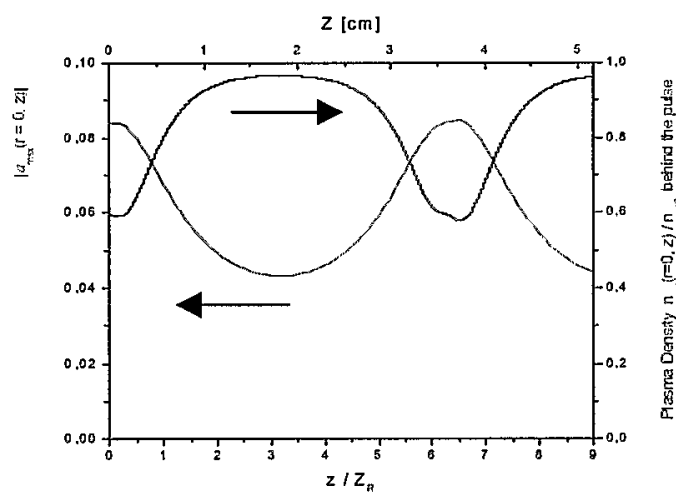

(a)

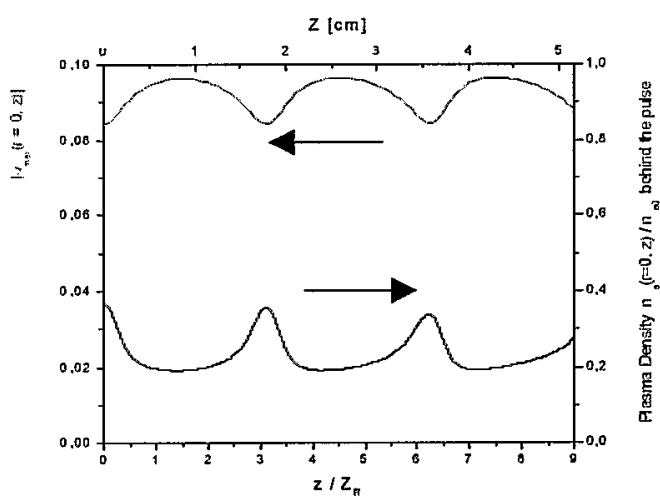

(b)

FIGURE 6. Distributions of the normalized laser pulse intensity and plasma density at the channel axis as functions of the length of propagation along the channel, a) mismatched condition in arbitrary plasma; b) matched condition in hydrogen plasma.
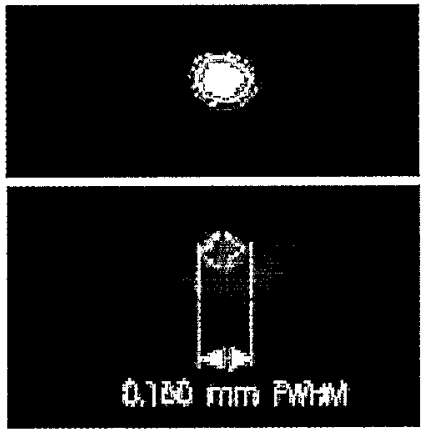

(a)

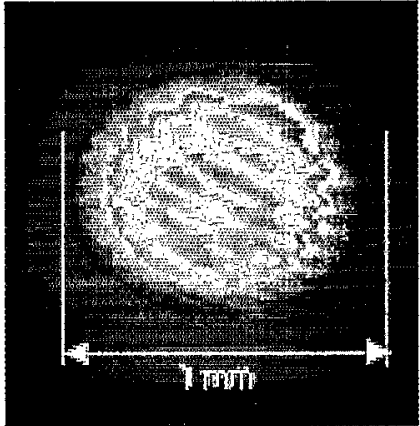

(b)

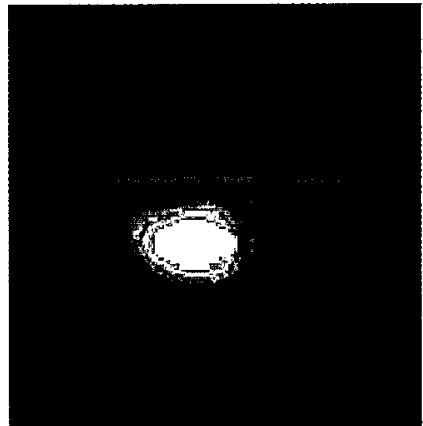

(c)

FIGURE 7. $\mathrm{CO}_{2}$ laser beam profiles:

a) at the focal point; b) $18 \mathrm{~mm}$ downstream from the focus in the free space propagation; c) at the exit of the plasma discharge with the capillary's entrance sited at the focal point. 


\section{LASER/ELECTRON BEAM COUNTER-PROPAGATION IN A CHANNEL}

Finally, we set up an experiment to explore counterpropagation of the $\mathrm{CO}_{2}$ laser and $60 \mathrm{MeV}$ electron beams inside the plasma channel.

We used Thomson x-ray scattering to demonstrate the time- and space-overlap of both beams. A 5-mm hole in the focusing parabolic mirror transmitted the backscattered $\mathrm{X}$-rays and the spent electron beam. X-rays are separated from electrons by a dipole magnet and detected by a Si diode placed after Be window outside the beamline.

An interaction of laser and e-beam in a free space or inside the "cold" capillary (without a discharge) results in a strong Thomson backscattering signal detected on the $\mathrm{Si}$ detector [6]. Energizing the discharge increases the signal up to eight times. We verified that the effect disappears each time when any contributor to the interaction is blocked (laser, or e-beam, or discharge). A phosphor screen placed on the path of the e-beam after the interaction simultaneously revealed that the beam acquires a strong angular spread and a part of it is stopped on the laser mirror. This accounts for the enhanced bremsstrahlung - the main contributor to the aforementioned increase of the x-ray signal, and indicates that channeling the laser beam through the plasma significantly modifies the electron beam's envelope.

We offer the following explanation for this effect:

A strong ponderomotive expulsion of plasma by a channeled $\mathrm{CO}_{2}$ laser pulse depletes the plasma's density inside the channel (Figure 5b). Due to the limited mobility of positive ions, the plasma inside the channel is not neutral, thus forming a positive column. The laser intensity defines a balance between the ponderomotive and chargeseparation forces. The electron beam injected inside this column experiences Coulomb attraction and, under certain condition, total "internal reflection" and guiding. This process resembles guiding electromagnetic waves inside the plasma channel. The difference is that the guiding of the electron beam is due to the Coulomb force of uncompensated space charge whereas the EM wave is guided by the radial gradient of a plasma density.

To formulate the conditions for laser-guided e-beam channeling, we use the correlation between the refraction angle $\theta$ with the incidence angle $\phi$ for an electron beam propagating inside the positively charged column of average density $N^{+}$and radius $R_{c h}$

$$
\theta=4 N^{+} r_{e} R_{c h}^{2} / \gamma \phi
$$

derived from the approach described in [7]. Disregarding ionic motion within the duration of the laser pulse we estimate

$$
N^{+} \approx n_{e 0} a_{0} \tau_{L} c / \pi R_{c h} .
$$

An electron beam with an intrinsic divergence

$$
\varphi=\varepsilon_{n} / \gamma \sigma_{b},
$$

where $\varepsilon_{n}$ is the normalized emittance, will be guided along the laser path when $\varphi \equiv \theta \leq \phi$. Combining Eq.(2-4) we arrive at the condition for channeling the electron beam 


$$
a_{0} \tau_{L} R_{c h} \geq \frac{\pi}{\gamma_{e} c}\left(\frac{\varepsilon_{n}}{2 \sigma_{b}}\right)^{2} \frac{1}{n_{e 0}} .
$$

For the conditions of the ATF experiment, the transient uncompensated charge-density produced by the laser with $a_{0} \approx 0.1$ is above $10^{16} \mathrm{~cm}^{-3}$ and Eq. (5) is easily satisfied.

E-beam guiding could be a useful effect that reduces tolerances to the e-beam/laser co-alignment. On the other hand, an unmatched electron beam might experience envelope oscillations similar to the laser beam, resulting in excessive e-beam losses.

All these findings and considerations can be important for ATF users' experiments, such as Thomson scattering in a plasma channel [1] or LWFA [8].

\section{CONCLUSIONS}

The following steps were taken along the path to putting plasma channel technology into service for ATF users' experiments:

- Conditions were established for producing cylindrically profiled plasma density on the scale of $\sim 10^{17} \mathrm{~cm}^{-3}$, and channeling of the intense $\mathrm{CO}_{2}$ laser beam was demonstrated.

- A capillary discharge setup was integrated into a laser/e-beam interaction cell; it proved compatible with the linac's high vacuum and laser optics.

- The plasma wake produced by an electron beam propagating through the channel was characterized using electron beam diagnostics.

- The channeled laser beam affects the propagation of the e-beam, suggesting new potentials for e-beam focusing, steering, and electron/laser synchronization.

\section{ACKNOWLEDGMENTS}

This study is supported by the U.S. Dept. of Energy under the Contract DE-AC0276CH00016, Japan-US and Japan-Israel Cooperative Grants in High Energy Physics.

\section{REFERENCES}

1. I.V. Pogorelsky, I. Ben-Zvi, X.J. Wang, and T. Hirose, Nucl. Instrum. \& Methods in Phys. Res. A 455, 176-180 (2000)

2. D. Kaganovich, P.V. Sasorov, A. Zigler, R. Burris, and Y. Ehrlich, Appl. Phys. Lett. 71, 2925-2927 (1997)

3. V. Yakimenko, et al., Phys. Rev. Lett. 91, 014802 (2003)

4. http:/www.bnl.gov/atf/Meetings/ATF2004/UserMeeting/KatsouleasTom.pdf

5. N.A. Bobrova, S.V. Bulanov, T.L. Razinkova, and P.V. Sasorov, Plasma Phys. Reps. 22, 349 (1996)

6. Pogorelsky, I.V., Ben-Zvi, I., Hirose, T., Kashiwagi, S., Yakimenko, V., Kusche, K., Siddons, P., Skaritka, J., Kumita, T., Tsunemi, A., Omori, T., Urakawa, J., Washio, M., Yokoya, K., Okugi, T., Liu, Y., He, P., and Cline, D., Phys. Rev. ST-AB 3, 090702 (2000)

7. P. Muggli, S. Lee, T. Katsouleas, R. Assman, F.J. Decker, M.J. Hogan, R. Iverson, P. Raimondi, R.H. Siemann, D. Walz, B. Blue, C.E. Clayton, E. Dodd, R.A. Fonseca, R. Hemker, C. Joshi, K.A. Marsh, W.B. Mori, and S. Wang, Phys. Rev. ST-AB 3, 091301 (2001)

8. W.D. Kimura, et al., "Pseudo-Resonant Laser Wakefield Acceleration Driven by $10.6 \mu \mathrm{m}$ Laser Light", to be published in IEEE Trans. Plasma Phys. 